Health \& Medicine | Jørgen Jahnsen

\section{A novel therapy could help patients with active Crohn's disease}

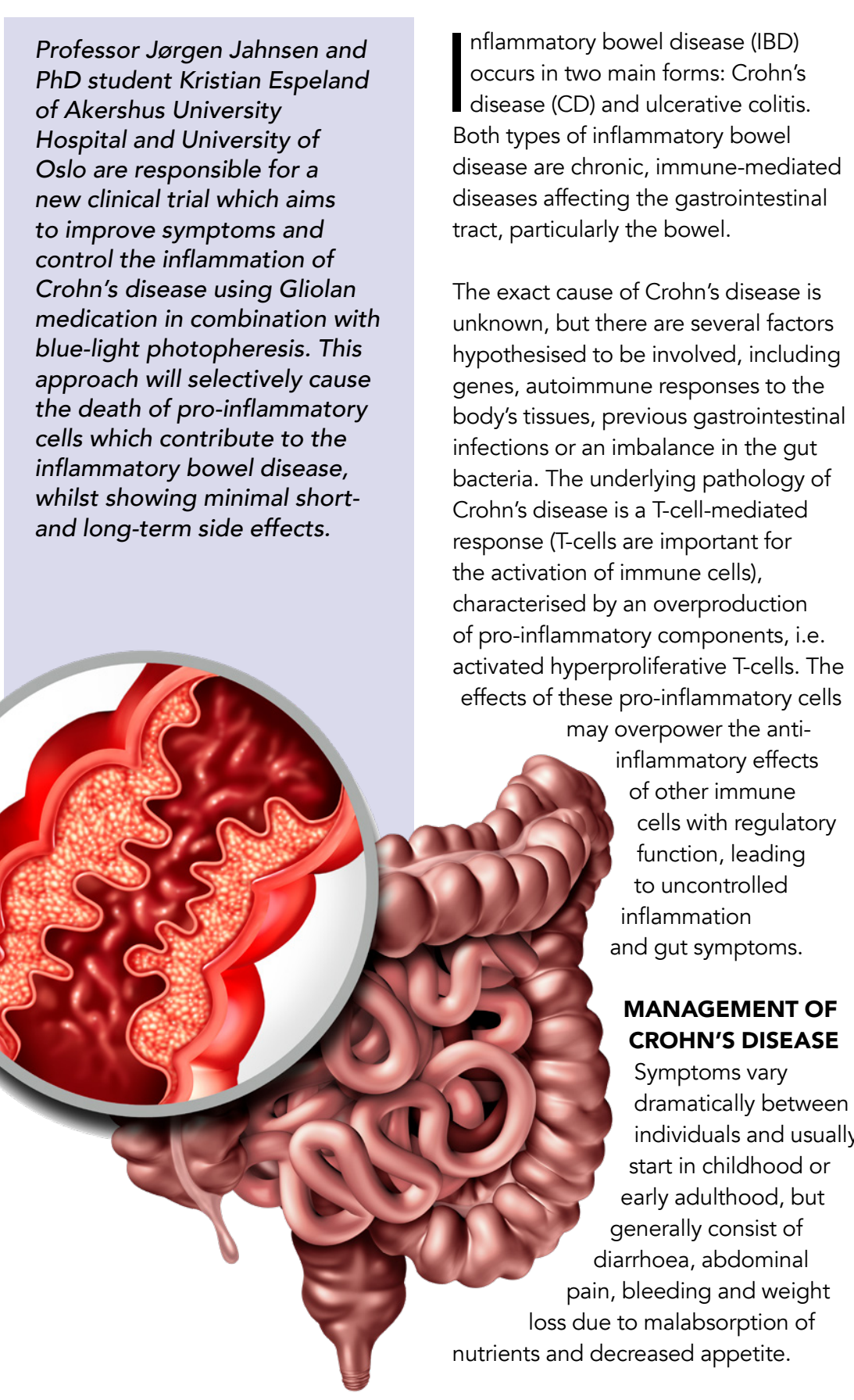

The symptoms may be constant or may flare up during episodic conts. There is also a risk of further complications requining surgery, such as and fistulae.

The Harvey Bradshaw Index (HBI) is often used to quantify disease activity in $C D$ based on symptoms and clinical signs and includes five factors: number of loose bowel movements, degree of abdominal pain, general wellbeing, presence of abdominal mass, and complications. In addition to assessing disease activity indices, the bowel inflammation can be evaluated by endoscopic examination and/ or measuring objective markers of intlammation such as faecal calprotectin and C-reactive protein.

The current goal of treatment is to minimising side effects from the drugs and allowing time for the tissue in the gut to heal. This is most often achieved using immunosuppressive and/or biological drugs, but these are not effective in all patients. Furthermore, there are risks involved with long-term use of these medications. Therefore, there is an urgent need to develop alternative therapies for $C D$, particularly for patients who are dependent on steroids, or who have a disease which is nonresponsive to available treatments.

Professor Jørgen Jahnsen and Kristian Espeland of Akershus University Hospita provide one such therapy-
PRINCIPLES OF PHOTOPHERESIS One promising technology for diseases, such as Crohn's disease, is extracorporeal photopheresis (ECP) technology. Indeed a previous publication in 2015 reported positive results from using ECP in patients with Crohn's disease. The process of photopheresis starts by withdrawing blood from the patient. Next, the white blood cells are separated from the red blood cells. These white blood cells are treated with a chemical whic makes them photosensitive, before being exposed to UV irradiation. Once they have been irradiated, the cells are eturned to the patient.

The exact mechanisms by which this process is clinically effective is not yet the photosensitistion agent induces T-cells to become apoptotic, or selfdestructing These dying cells are take up by other cells in the immune system and, along with the chemicals released as a result of the process, increase the activity of regulatory immune cells. Ultimately, ECP seems to dampen down inflammation without lowering the body's immunity against other infections, resulting in better long-term outcomes for patients.

However, a major disadvantage of this approach is that it kills both light exposure. Thus, the is light exposure. Thus, there is an urgent effective alternative.

One such alternative, which will be used by the investigators, is a two-ste process called BLUE-PIT. This process uses a drug called Gliolan, which is often employed to visualise tumours prior to surgery as it causes them to glow fluorescent red. Gliolan leads to the accumulation of protoporphyrin X (PpIX), a potent photosensitizer, in hyperproliferative cells, such as the activated T-cells in Crohn's disease. Blue light $(405 \mathrm{~nm})$ is the most effective wavelength for PolX activation which consequently induces apoptosis, mainly in activated T-cells. The treated cells are transferred back to the patient and can induce furt tor

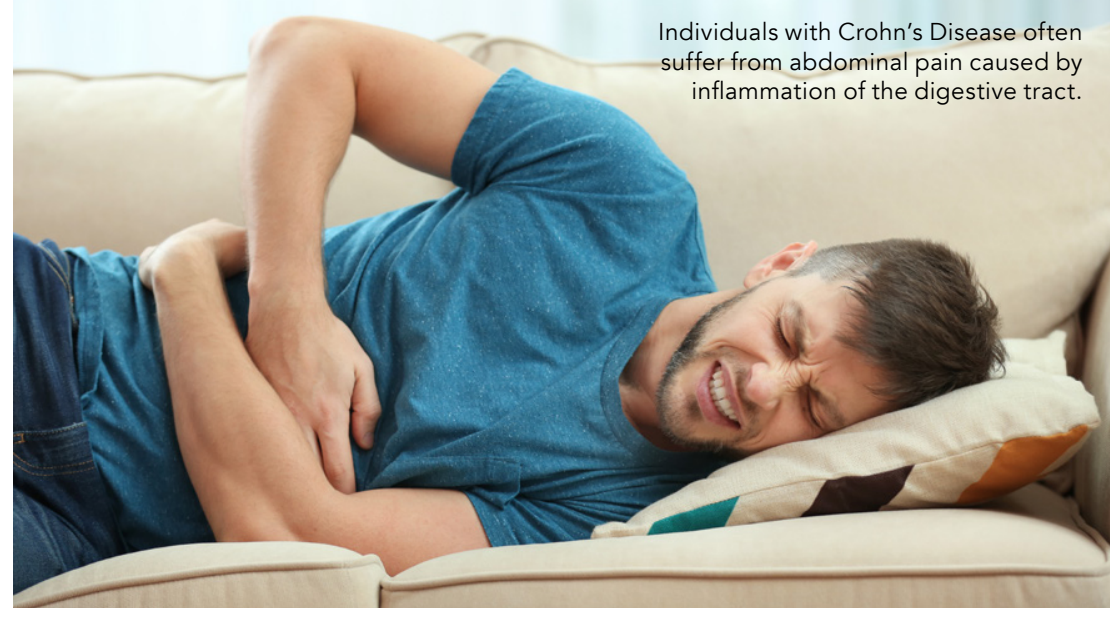

EVIDENCE FROM PRECLINICAL TRIALS

is also a reduced risk of carcinogenesis Prof Jahnsen and his team have already likelihood that the therapy will induce an undertaken preclinical trials to support this T-cells produce about 15 -fold more Gliolan-induced PpIX than normal

T-cells, meaning that they are selectively destroyed after light irradiation, leaving normal immune cells undamaged. There

Blue light has been demonstrated to be inducing apoptosis in T-cells, and ECP. appears to be well tolerated and have fewer side effects than traditionally used immunosuppressants. Furthermore,

One promising technology for the treatment of T-cell-mediated diseases, such as Crohn's disease, is extracorporeal photopheresis (ECP) technology.

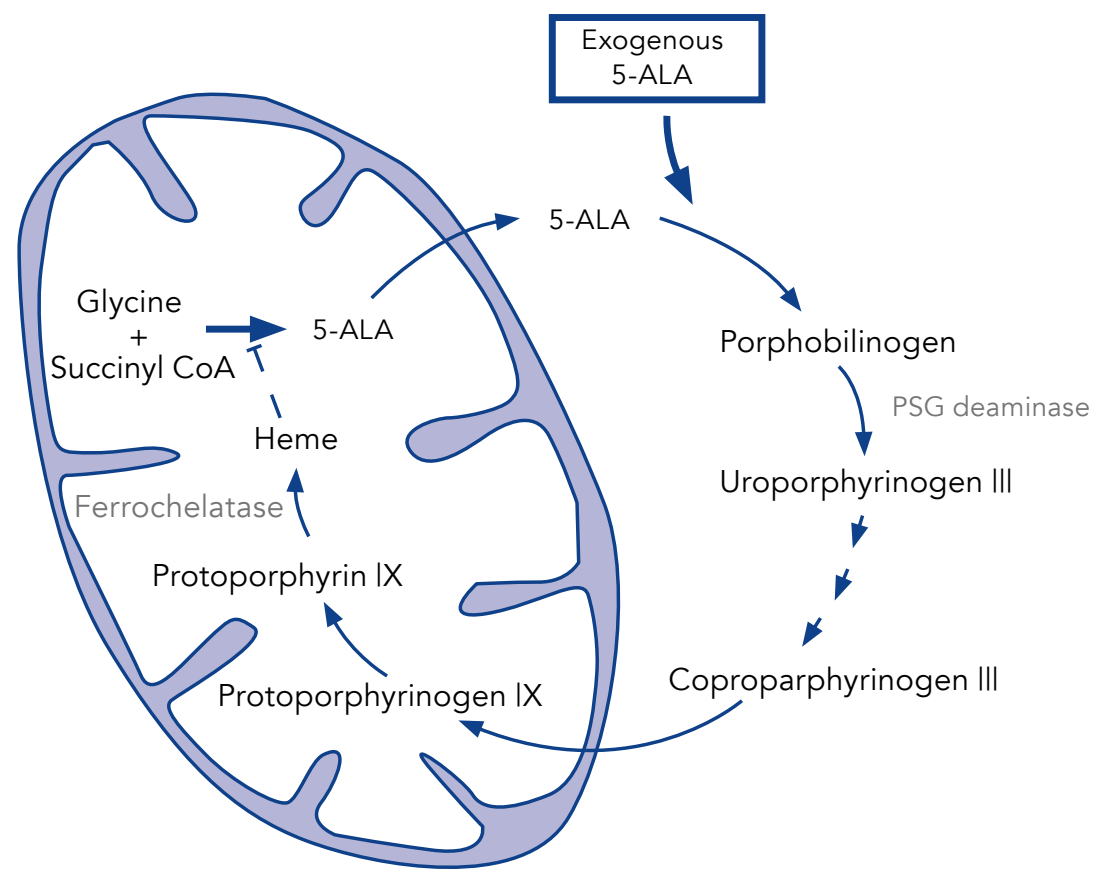
Exogenous 5 pathway.
pats 


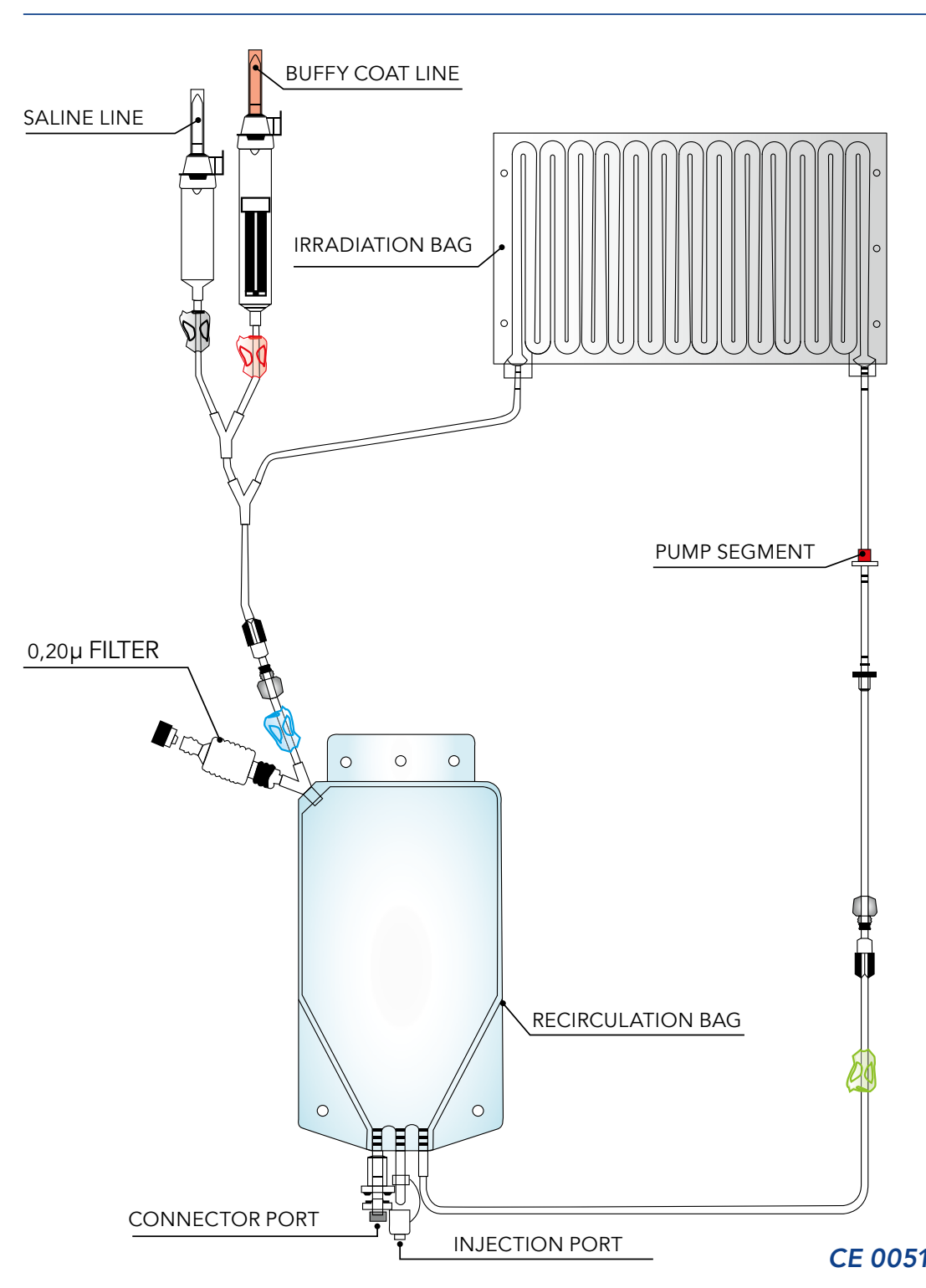

The research team employs swo-step photopheresis to separate white
cells and consequenty treat the former with Gilolan and blue light.

Ultimately, ECP seems to dampen down inflammation without lowering the body's immunity against other infections, resulting in better long-term outcomes for patients.

as Gliolan will only be given once per treatment, this reduces the potential for side-effects and the visible blue ight source has not

Using a one-day treatment, which appears to be as effective as the standard two-day treatment, will greatly reduce costs as well as the burden for patients. Both these factors will nake the therapy more attractive for
The study proposed by Prof Jahnsen is running from September 2019 until February 2022. The study is a proofof-concept pilot with ten included patients, where every patient will get active treatment. The use of Gliolan in combination with blue-light photopheresis is a first-in-human trial. The investigators will assess efficacy, safety and tolerability after single in combination with blue ligh Crohn's disease. All patients will Cronn's disease. Al patients will en weeks with evaluation a weeks for any effect is observed during week 13 , the study is eligible for an extension for up to 12 months.

Primary endpoints include clinica response as measured by a reduction of three or more points in $\mathrm{HBI}$ score. This reduction in symptom score must ideally be sustained after treatment. Safety and tolerability will be monitore through frequency, seriousness and

intensity of adverse events.

Secondary endpoints include

endoscopic improvement, quality of C- questive protein, aecal calprotectin, action (differences in T-cells and other cells before and after treatment). The researchers will also be looking for clinical remission throughout the study.

The overall aim of the study is to investigate whether this kind of photopheresis is safe and presents an option for a larger randomised

controlled trial in the future. In addition, Prof Jahnsen and his team will consider whether photopheresis provides

potential therapeutic benefits for other diseases, such as other T-cell-mediated diseases or patients already receiving

\section{FUTURE IMPLICATIONS}

Although there are some risks

ssociated with the study, Prof Jahnsen

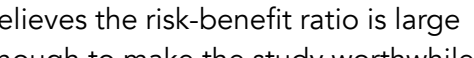
ff there are improvements to symptoms experienced by patients with Crohn's disease, in addition to the therapy being safe and tolerated, this supports further studies being done in the future. Living with IBD may negatively affect patients health-related quality of life, therefore therapies to manage symptoms have benefits for physical and mental health. Validation of a new, clinically and financially viable therapeutic has the potential to improve the quality of life for the Eree min in paple in the USA and worldwide living with Crohn's of others

\section{Behind the Research}

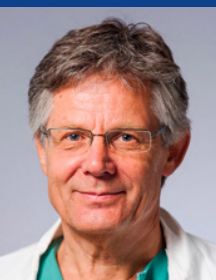

Dr Jørgen

Jahnsen

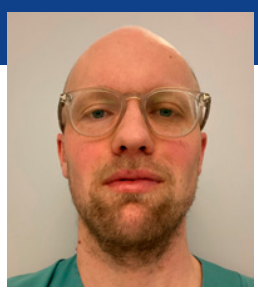

Dr Kristian Espeland
Research Objectives

Prof Jahnsen and PhD student Espeland conduct a clinical trial on Gliolan medication in combination with blue-light photopheresis in patients with active Crohn's disease.

\section{Detail}

Jørgen Jahnsen

itetssykehus $\mathrm{HF}$ Postboks 4950 Nydalen 0424 Oslo

Bio
Jørgen Jahnsen is professor of medicine and gastroenterology and holds a PhD on bone metabolism and bowel disease (IBD). He has broad

research experience in the field of
gastroenterology. National Coordinator
(NC) of several phase II/III clinical trials.
Principal Investigator (PI) on the project.
Kristian Espeland is a PhD candidate
at the University of Solo and Sponsor
Principal Investigator (SPI) on the project.
Funding
South-Eastern Norway Regional
Health Authority

Collaborators
Qian Peng (Professor, MD, PhD) Eidi Christensen (Associate Professor, MD, PhD), Astrid Aandahl (MD), Andreas Ulvær, Kristian Espeland (MD, PhD student), Sagar Darvekar (PhD), Andrius Kleinauskas ( $\mathrm{PhD}$ candidate), Morten Oksvold (PhD), Trond Warloe (MD, PhD), Petras Juzenas (PhD), Vlad Health Authority

\section{References}

Jahnsen, J., Espeland, K. et al. (2019). Extracorporeal photopheresis of patients with Crohn's disease using 5-aminolevulinic
protocol, unpublished.

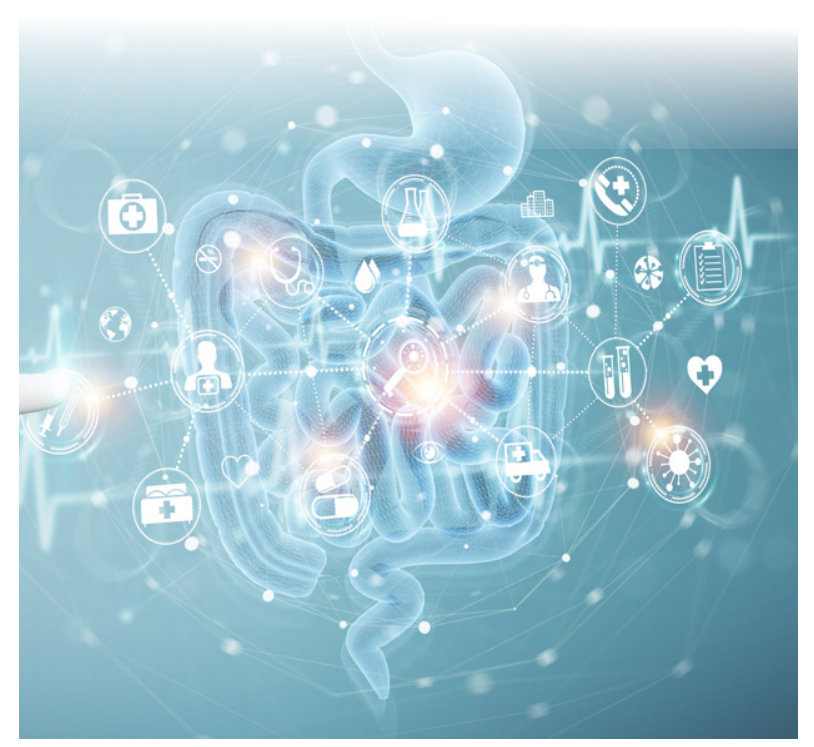

\section{Personal Response}

What is the most promising result so far?

II It is far too early to conclude after one patient. What we have seen is that Glolan-based photopheresis is seems safe. We look very much forwat to include more patients and maybe try the treatment on other diseases in later studies, for example other immunemediated disorders such as rheumatic (rheumatoid arthritis, psoriatic arthritis, ankylosing spondylitis) and photopheresis works might have an effect by using this

Gliolan-based photopheresis with blue light.

AKERSHUS UNIVERSITY HOSPITAL

$\mathrm{UiO}:$ University of Oslo 